\title{
TRANSFORMAÇÃO DA MATÉRIA ORGÂNICA E DO NITROGÊNIO DURANTE A COMPOSTAGEM DA FRAÇÃO SÓLIDA DO CHORUME BOVINO(1)
}

\author{
Luis Miguel Brito ${ }^{(2)}$, Ana Luisa Amaro(3), Isabel Mourão ${ }^{(4)} \&$ \\ João Coutinho(5)
}

\section{RESUMO}

\begin{abstract}
A fração sólida do chorume (FSC) de duas explorações de pecuária leiteira intensiva (FSC1 e FSC2, recolhidas em fossa aberta e fechada, respectivamente) foi compostada com e sem adição de palha, em pilhas estáticas e em pilhas com revolvimento. A temperatura aumentou rapidamente na FSC1, que foi recolhida mais lentamente, até a máxima diária de $68^{\circ} \mathrm{C}$, registrada na pilha estática com palha. No entanto, a FSC2 não alcançou temperatura superior a $51^{\circ} \mathrm{C}$ em qualquer uma das pilhas. A mistura de palha com a FSC aumentou a temperatura no início da compostagem em todas as pilhas, mas as taxas de mineralização da matéria orgânica (MO) não aumentaram. Nas pilhas com revolvimento, a MO potencialmente mineralizável aumentou em comparação com as pilhas estáticas, mas a concentração de $\mathrm{N}$ nos compostos finais foi mais elevada nas pilhas sem revolvimento, sugerindo que o revolvimento pode ter aumentado as perdas de $\mathrm{N}$. A relação C/N da FSC diminuiu de forma semelhante em todas as pilhas, desde um valor inicial de 27 ou superior até o valor de 11 a 13 no final da compostagem. A baixa temperatura, a baixa relação $\mathrm{C} / \mathrm{N}$ e a baixa concentração de $\mathrm{NH}_{4}{ }^{+} \mathrm{em}$ combinação com o aumento da concentração de $\mathrm{NO}_{3}{ }^{-}$dos compostos finais indicaram que estes estavam estabilizados. A elevada concentração de MO (720$820 \mathrm{~g} \mathrm{~kg}^{-1}$ ) e de $\mathrm{N}$ total (30-44 $\mathrm{g} \mathrm{kg}^{-1}$ ) e a baixa condutividade elétrica (inferior a $100 \mathrm{mS} \mathrm{m}^{-1}$ ) sugerem que os compostos da FSC podem ser utilizados como corretivos orgânicos do solo com benefícios agronômicos e ambientais. No entanto,
\end{abstract}

\footnotetext{
(1) Recebido para publicação em julho de 2007 e aprovado em julho de 2008.

(2) Professor do Instituto Politécnico de Viana do Castelo. Escola Superior Agrária, Refóios 4990-706 Ponte de Lima, Portugal. Email: miguelbrito@esapl.pt

(3) Engenheira-Agrônomo do Instituto Politécnico de Viana do Castelo. Escola Superior Agrária, Portugal. E-mail: anafernandes@portugalmail.com

(4) Professora do Instituto Politécnico de Viana do Castelo. Escola Superior Agrária, Portugal. E-mail: isabelmourao@esapl.pt

(5) Professor da Universidade de Trás-os-Montes e Alto Douro - UTAD. 5001-911 Vila Real, Portugal. E-mail: j_coutin@utad.pt
} 
é necessário continuar a investigar para garantir a higienização do composto, porque a FSC2 não alcançou temperaturas termófilas durante a compostagem.

Termos de indexação: adubação orgânica, composto, corretivos orgânicos, mineralização, pecuária leiteira.

\title{
SUMMARY: TRANSFORMATION OF ORGANIC MATTER AND NITROGEN DURING COMPOSTING OF THE SOLID FRACTION OF CATTLE SLURRY
}

\begin{abstract}
Cattle slurry solid fraction (FSC1 and FSC2, collected from open and closed sinkholes, respectively) was collected from two dairy farms and composted with and without straw, in static and in turned piles. Thermophilic temperatures were attained soon after separation of FSC1, which was collected at lower rate, and increased to a daily maximum temperature of $68{ }^{\circ} \mathrm{C}$ in the static pile with straw. However, the temperature in the FSC2 piles did not reach temperatures above $51^{\circ} \mathrm{C}$. Straw addition to slurry solid fraction contributed to raise temperatures in all piles, but did not increase organic matter (OM) mineralization rates. Maximum mineralizable OM was higher in the turned than in static piles but the final $N$ compost content was higher in static piles, suggesting that revolving can increase $N$ losses. The C/N ratio declined from over 27 to a value of 11-13 towards the end of composting and followed a similar pattern for all compost treatments. Stabilized compost was obtained from raw $S F$ feedstock as indicated by the low compost temperature, low C/N ratio and the low $\mathrm{NH}_{4}{ }^{+}$content, besides higher $\mathrm{NO}_{3}{ }^{-}$concentrations. The high concentration of $\mathrm{OM}$ $\left(720-820 \mathrm{~g} \mathrm{~kg}^{-1}\right)$ and total $\mathrm{N}\left(30-44 \mathrm{~g} \mathrm{~kg}^{-1}\right)$ in final composts in addition to a low electrical conductivity (below $100 \mathrm{mS} \mathrm{m} \mathrm{m}^{-1}$ ) suggests that $\mathrm{SF}$ composts may be useful as soil amendments with agronomic and environmental advantages. However, further research is needed to assure compost hygienization since the FSC2 did not reach thermophilic temperatures during composting.
\end{abstract}

Index terms: compost, dairy cattle, mineralization, organic amendments, organic fertilizer.

\section{INTRODUÇÃO}

A separação do chorume de bovinos leiteiros (esterco de gado leiteiro) em uma fração sólida do chorume (FSC) e uma fração líquida tornou-se uma técnica de uso crescente na gestão deste efluente da pecuária leiteira intensiva (Ford \& Fleming, 2002). Esta técnica introduz uma melhoria nas propriedades de manejo do chorume, reduzindo a quantidade de matéria orgânica dos líquidos e concentrando a matéria seca na fração sólida, que pode ser compostada para produzir um fertilizante rico em matéria orgânica e nutrientes.

Durante a compostagem, a atividade metabólica da população microbiana resulta na produção de calor e no aumento de temperatura. O aquecimento e 0 arrefecimento do material orgânico fornecem informação sobre sua atividade biológica e seu grau de decomposição. Stentiford (1996) sugeriu que temperaturas entre 35 e $40^{\circ} \mathrm{C}$ maximizam a diversidade microbiana no processo de compostagem. Com temperaturas superiores a $40^{\circ} \mathrm{C}$, a nitrificação e a desnitrificação e, conseqüentemente, a produção e emissão de $\mathrm{N}_{2} \mathrm{O}$ são suprimidas (Hellmann et al.,
1997), porque a atividade das bactérias autotróficas nitrificantes desaparece (Myers, 1975). Em contrapartida, a temperatura ótima para os fungos termófilos e os actinomicetos, responsáveis pela degradação de lignina, varia entre 40 e $50^{\circ} \mathrm{C}$ (Hellmann et al., 1997). As bactérias termófilas possuem uma temperatura ótima variável entre 45 e $50{ }^{\circ} \mathrm{C}$ ou superior (Golueke, 1977). Tchobanoglous et al. (1993) observaram que Salmonella sp. e Escherichia coli podem ser destruídas em 15 a 20 min quando expostas a $60^{\circ} \mathrm{C}$, ou durante uma hora, a $55^{\circ} \mathrm{C}$. Com essa valores de temperatura, o processo de compostagem pode eliminar todos os organismos patogênicos e as sementes viáveis de infestantes, preocupação fundamental na aplicação direta do chorume ao solo. Contudo, quando a umidade do material orgânico em compostagem excede 70 \%, o que é frequente com a FSC (Brito et al., 2006), elevadas temperaturas podem não ser atingidas devido à restrição na entrada de $\mathrm{O}_{2}$ para materiais em compostagem.

A eficácia do processo de compostagem, a taxa de atividade metabólica e a temperatura são condicionadas pelas características físicas da FSC, como a porosidade 
e a umidade. A FSC é constituída por um material fibroso, com porosidade, o que, dentro de certos limites, permite a entrada de ar na pilha em compostagem (Miller et al., 1989). A entrada de ar aumenta pela ação do fluxo ascendente de ar provocado pela produção de calor durante o processo de compostagem (Oenema et al., 2001).

Os processos de volatilização da amônia e de desnitrificação são responsáveis pelas maiores perdas de N durante a compostagem. Eghball et al. (1997) e Tiquia \& Tam (2000) encontraram na literatura referências a perdas de N entre 21 e $77 \%$. Raviv et al. (2004) relataram perdas semelhantes (16-74\%), e concluíram que a maioria das perdas de $\mathrm{N}$ era causada pela volatilização de $\mathrm{NH}_{3}$. As perdas de $\mathrm{N}$ durante o processo de compostagem aumentam com a temperatura e a intensidade com que se realizam as trocas gasosas com o exterior, com a diminuição da relação $\mathrm{C} / \mathrm{N}$ e com o aumento do $\mathrm{pH}$ (Sommer \& Moller, 2000; Raviv et al., 2004), mas diminuem com a cobertura das pilhas (Webb et al., 2001; Sommer, 2001; Chadwick, 2005; Hansen, et al., 2006).

Há diversos estudos realizados com a FSC de suínos (Tiquia et al., 1997; Møller et al., 2000; Fukumoto et al., 2003; Huang et al., 2006; Ros et al., 2006). No entanto, as transformações da matéria orgânica e do $\mathrm{N}$ durante a compostagem da FCS de bovinos leiteiros não são bem conhecidas. Neste estudo, comparase a evolução de características físicas e químicas no processo de compostagem da FSC de duas explorações de pecuária leiteira intensiva, em pilhas de compostagem com e sem arejamento, ou adição de palha, com o objetivo de obter um produto final estabilizado, de elevada qualidade, rico em $\mathrm{N}$ e em MO.

\section{MATERIAIS E MÉTODOS}

Construíram-se oito pilhas de compostagem, quatro com a FSC recolhida de uma exploração leiteira com o chorume armazenado numa fossa aberta e quatro com a FSC de outra exploração com fossa fechada, ambas localizadas em Vila do Conde no NW de Portugal. As máquinas separadoras utilizadas na extração da FSC baseavam-se na pressão provocada por uma rosca sem-fim que impulsiona os sólidos para a extremidade frontal da máquina, enquanto a fração líquida atravessa um crivo que rodeia a rosca. Na primeira exploração, o rendimento da máquina foi inferior $\left(1 \mathrm{~m}^{3} \mathrm{~h}^{-1}\right)$ em comparação com o da segunda $\left(3 \mathrm{~m}^{3} \mathrm{~h}^{-1}\right)$. Metades das pilhas foram misturadas com palha de cevada ( $21 \%$ em peso do material seco, $7 \%$ em peso fresco). Em cada duas pilhas semelhantes, uma sofreu cinco revolvimentos e a outra não teve qualquer revolvimento (Quadro 1).

O revolvimento realizou-se com a pá frontal de um trator aos 14, 28, 56, 84 e 133 dias de compostagem. As pilhas iniciaram-se com 1,7 m de altura ao ar livre, sobre uma tela de cobertura do solo de polietileno, para impedir a entrada de terra durante o revolvimento, e foram cobertas com uma tela de polipropileno não tecido, tipo geotextil (Toptex), que impediu a infiltração da água da chuva, mas possibilitou as trocas gasosas com o exterior.

As temperaturas no centro das pilhas e do ambiente exterior foram medidas em cada minuto com termístores (tipo ST1, Delta-T Devices). Os valores médios horários foram registrados com um registrador de dados (Delta-T Devices) e, com base nesses registros, calcularam-se as temperaturas médias diárias.

Realizaram-se oito coletas de quatro amostras por cada pilha nos seguintes dias de compostagem: 0,14 , $28,56,84,112,140$ e 168 . Recorreram-se às normas européias (CEN, 1999) para a determinação das seguintes características: umidade, com base em $50 \mathrm{~g}$ de material original (EN 13040); pH por potenciometria, utilizando extratos de $60 \mathrm{~cm}^{3}$ de amostra por $300 \mathrm{~mL}$ de água (EN 13037); condutividade elétrica dos extratos aquosos obtidos de acordo com a norma do $\mathrm{pH}(1+5, \mathrm{v} / \mathrm{v})$, após filtragem (EN 13038) com um condutivímetro; MO por calcinação em mufla, a $550{ }^{\circ} \mathrm{C}$ durante $4 \mathrm{~h}$ (EN 13039); e N (N) Kjeldahl modificado (EN 13654).

Quadro 1. Modalidades de compostagem da fração sólida do chorume (FSC) recolhido de fossa aberta (FSC1) e fechada (FSC2)

\begin{tabular}{|c|c|c|c|}
\hline Origem da FSC & Composição & Revolvimento & Símbolo \\
\hline \multirow[t]{2}{*}{ FSC1 } & FSC & $\begin{array}{l}\text { Com revolvimento } \\
\text { Sem revolvimento }\end{array}$ & $\begin{array}{l}\text { FSC1 (CR) } \\
\text { FSC1 (SR) }\end{array}$ \\
\hline & FSC + palha & $\begin{array}{l}\text { Com revolvimento } \\
\text { Sem revolvimento }\end{array}$ & $\begin{array}{l}\mathrm{FSC} 1+\mathrm{P}(\mathrm{CR}) \\
\mathrm{FSC} 1+\mathrm{P}(\mathrm{SR})\end{array}$ \\
\hline \multirow[t]{2}{*}{ FSC2 } & FSC & $\begin{array}{l}\text { Com revolvimento } \\
\text { Sem revolvimento }\end{array}$ & $\begin{array}{l}\text { FSC2 }(\mathrm{CR}) \\
\text { FSC2 }(\mathrm{SR})\end{array}$ \\
\hline & FSC + palha & $\begin{array}{l}\text { Com revolvimento } \\
\text { Sem revolvimento }\end{array}$ & $\begin{array}{l}\mathrm{FSC} 2+\mathrm{P}(\mathrm{CR}) \\
\mathrm{FSC} 2+\mathrm{P}(\mathrm{SR})\end{array}$ \\
\hline
\end{tabular}


A relação $\mathrm{C} / \mathrm{N}$ foi calculada pelo quociente entre o teor de $\mathrm{C}$ e o de $\mathrm{N}$. O teor de $\mathrm{C}$ total, destinado ao cálculo da relação $\mathrm{C} / \mathrm{N}$, foi determinado pela fração entre o teor da matéria orgânica e a constante 1,8 (Gonçalves \& Baptista, 2001). As perdas de $\mathrm{MO}\left(\mathrm{g} \mathrm{kg}^{-1}\right)$ foram estimadas pela fórmula:

$$
\text { 1000-1000 [X1 (1000-X2)] / [X2 (1000-X1)] }
$$

em que X1 e X2 representam as cinzas $\left(\mathrm{g} \mathrm{kg}^{-1}\right)$, respectivamente, no início e no fim de cada período de compostagem (Paredes et al., 2000).

A mineralização da MO foi estimada pelas perdas de $\mathrm{MO}$ e obedeceu a uma cinética de $1^{\mathrm{a}}$ ordem, expressa pela seguinte equação:

$$
\mathrm{MO}_{\mathrm{m}}=\mathrm{MO}_{0}\left(1-\mathrm{e}^{-\mathrm{kt}}\right)
$$

em que $\mathrm{MO}_{\mathrm{m}}$ representa a matéria orgânica $\left(\mathrm{g} \mathrm{kg}^{-1}\right)$ mineralizada no tempo $\mathrm{t}$ (dia); $\mathrm{MO}_{0}$, a matéria orgânica potencialmente mineralizável $\left(\mathrm{g} \mathrm{kg}^{-1}\right)$, e $\mathrm{k}$, a taxa de mineralização.

Parte das amostras foram congeladas imediatamente após a colheita, para a determinação do teor de $\mathrm{N}$ mineral, após extração com KCl $2 \mathrm{~mol} \mathrm{~L}^{-1}$ (1:5) por espectofotometria de absorção molecular (Houba et al., 1995) em autoanalisador de fluxo segmentado, sendo o teor de $\mathrm{N}$ amoniacal determinada pela reação de Berthelot e a de $\mathrm{N}$ nítrico por meio do reagente de Griess-Ilosvay, após redução em coluna de Cd.

Utilizaram-se o método de Levenberg-Marquardt na análise de regressão não-linear da mineralização da MO e o teste Duncan para comparar as médias das propriedades químicas analisadas, recorrendo-se ao programa SPSS, versão 15.0. (SPSS Inc.).

\section{RESULTADOS}

A fase termófila $\left(>50^{\circ} \mathrm{C}\right)$ da compostagem iniciouse pouco tempo depois da construção das pilhas com a FSC1 da fossa de chorume aberta e prolongou-se por um mês, alcançando temperatura superior a $60^{\circ} \mathrm{C}$ nas pilhas que incluíram palha (Figura 1). Contudo, as temperaturas durante a compostagem da FSC2 proveniente da fossa de chorume fechada foram inferiores, alcançando apenas, e só pontualmente, temperatura de $50{ }^{\circ} \mathrm{C}$ nas pilhas com revolvimento. A adição de palha à FSC contribuiu para aumento de temperatura, mais evidente nas pilhas sem revolvimento em comparação com as pilhas com revolvimento.

A máxima temperatura $\left(68^{\circ} \mathrm{C}\right)$ foi registrada na pilha da FSC1 com palha e sem revolvimento aos 17 dias de compostagem. Nas pilhas com revolvimento, a temperatura máxima $\left(66^{\circ} \mathrm{C}\right)$ foi atingida com a FSC1 com palha. Aproximadamente após dois meses de compostagem, as temperaturas diminuíram gradualmente até um valor semelhante ao da temperatura ambiente, alcançado entre o quarto e o sexto mês de compostagem.

A FSC foi recolhida com uma umidade de $75 \%$ (FSC1) e de $76 \%$ (FSC2) enquanto a palha possuía $14 \%$ de umidade. A compostagem da FSC decorreu com valores de umidade geralmente superiores a $70 \%$ naquelas sem palha, e a $60 \%$ naquelas com palha.
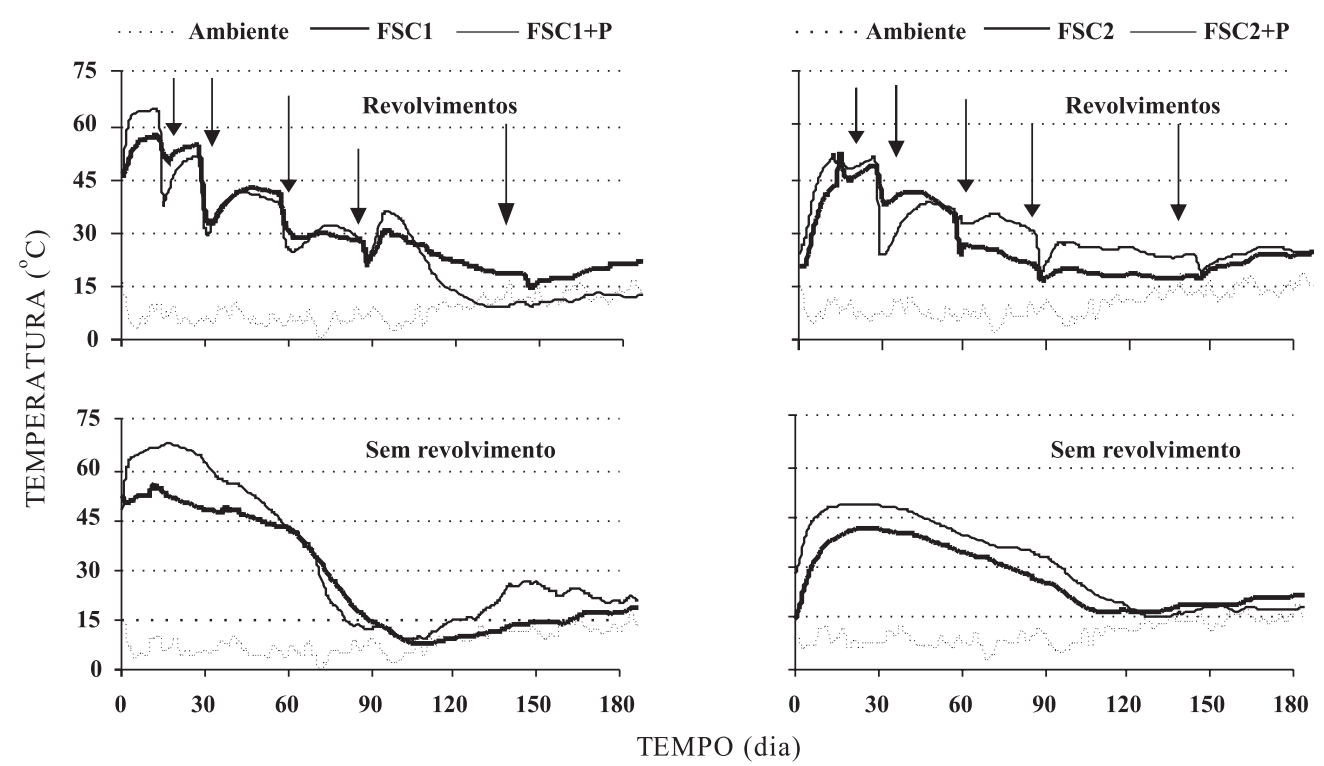

Figura 1. Evolução da temperatura média do interior das pilhas e do ambiente exterior, em função do tempo de compostagem da fração sólida do chorume (FSC). As pilhas incluíram diferentes origens (FSC1 e FSC2, recolhidos de fossa aberta e fechada, respectivamente), com revolvimento e sem revolvimento, sem e com palha (P). 
A exceção verificou-se na pilha estática da FSC1 com palha onde, devido a um fenômeno de secagem em alguns locais da pilha, foram colhidas amostras com umidade variável entre 45 e $64 \%$. A umidade da FSC1 foi ligeiramente inferior à da FSC2 durante a compostagem, com maior diferença nas pilhas estáticas com palha. O revolvimento das pilhas não contribuiu para redução da umidade, mas verificouse diminuição de $7 \%$ na média da umidade das pilhas com palha, em comparação com as pilhas sem palha. Para o conjunto dos oito tratamentos, o valor de $\mathrm{pH}$ foi sempre alcalino, variando entre o máximo de 9 e o mínimo de 7,2 (Quadro 2). A condutividade elétrica dos extratos aquosos variou entre 46 e $110 \mathrm{mS} \mathrm{m}^{-1}$ para o conjunto das pilhas e foi geralmente superior na FSC2, em comparação com a FSC1 (Quadro 2).

O teor de matéria orgânica (MO) no início foi, respectivamente, de 890, 899 e $822\left(\mathrm{~g} \mathrm{~kg}^{-1}\right)$ para a FSC1, a FSC2 e a palha. O teor de MO diminuiu em todas as pilhas até um valor mínimo de $722 \mathrm{~g} \mathrm{~kg}^{-1}$, 168 dias após o início da compostagem na pilha com a FSC1 e com o revolvimento.

As perdas de MO (Figura 2) sugerem duas fases de mineralização. A primeira, com um forte declive, indica a rápida mineralização do substrato mais facilmente mineralizável e ocorreu nos primeiros dois meses de compostagem. A segunda fase revela uma taxa inferior de mineralização da MO, ao restarem só os constituintes mais resistentes à decomposição.
Mais de metade da MO das pilhas com revolvimento foi mineralizada num período de dois meses de compostagem, mais rapidamente nas pilhas com a FSC2, em que mais de $400 \mathrm{~g} \mathrm{~kg}^{-1}$ foram mineralizadas em menos de um mês (Figura 2). No final da compostagem, a mineralização ultrapassou os $600 \mathrm{~g} \mathrm{~kg}^{-1} \mathrm{em}$ todas estas pilhas com revolvimento, valor que não foi alcançado em qualquer uma das pilhas sem revolvimento. As taxas de mineralização diminuíram sempre com adição de palha, mas de forma significativa apenas na pilha da FSC1 sem revolvimento.

$\mathrm{O}$ teor de $\mathrm{N}$ na matéria seca aumentou com a diminuição do teor da MO (Figura 3) de forma quadrática, entre o mínimo de $18 \mathrm{~g} \mathrm{~kg}^{-1}$, no início da compostagem na FSC1, e de $14 \mathrm{~g} \mathrm{~kg}^{-1}$ na FSC2, até ao máximo absoluto de $44 \mathrm{~g} \mathrm{~kg}^{-1}$ no final da compostagem, registrado numa das amostras da pilha estática da FSC2 sem palha. Esta figura 3 revela a tendência para o aumento da concentração de $\mathrm{N}$ nas pilhas estáticas em comparação com as pilhas com revolvimento. A relação C/N diminuiu de 27 nas pilhas com a FSC1 e de 37 nas pilhas com a FSC2, no início da compostagem, até um valor mínimo de 11 no final da compostagem (Figura 4). A acentuada decomposição da MO na fase inicial da compostagem foi responsável pelo rápido aumento do teor de $\mathrm{N}$ nesta fase e pela acentuada diminuição da relação $\mathrm{C} / \mathrm{N}$, para valores inferiores a 20 em menos de um mês de compostagem.

Quadro 2. Valores de pH e condutividade elétrica das pilhas de compostagem com diferentes dimensões de origem (FSC1 e FSC2, recolhidos de fossa aberta e fechada, respectivamente), com revolvimento (CR) e sem revolvimento (SR), sem palha e com palha (P) (médias \pm erro-padrão)

\begin{tabular}{|c|c|c|c|c|c|c|c|c|}
\hline \multirow{2}{*}{$\begin{array}{l}\text { Pilhas de } \\
\text { compostagem }\end{array}$} & \multicolumn{8}{|c|}{ Tempo (dia) } \\
\hline & $\mathbf{0}$ & 14 & 28 & 56 & 84 & 112 & 140 & 168 \\
\hline & \multicolumn{8}{|c|}{$\mathrm{pH}$} \\
\hline CR - FSC1 & $7,9 \pm 0,1$ & $8,6 \pm 0,2$ & $8,9 \pm 0,1$ & $8,7 \pm 0,1$ & $8,4 \pm 0,1$ & $8,1 \pm 0,1$ & $7,9 \pm 0,2$ & $7,7 \pm 0,1$ \\
\hline $\mathrm{CR}-\mathrm{FSC} 1+\mathrm{P}$ & $7,7 \pm 0,1$ & $8,8 \pm 0,2$ & $8,9 \pm 0,1$ & $8,4 \pm 0,1$ & $8,3 \pm 0,1$ & $7,7 \pm 0,1$ & $7,4 \pm 0,1$ & $7,2 \pm 0,1$ \\
\hline $\mathrm{CR}-\mathrm{FSC} 2$ & $8,8 \pm 0,1$ & $9,0 \pm 0,1$ & $8,9 \pm 0,1$ & $9,0 \pm 0,1$ & $8,4 \pm 0,2$ & $8,2 \pm 0,1$ & $8,2 \pm 0,1$ & $8,5 \pm 0,1$ \\
\hline $\mathrm{CR}-\mathrm{FSC} 2+\mathrm{P}$ & $8,4 \pm 0,1$ & $8,8 \pm 0,1$ & $8,9 \pm 0,1$ & $8,6 \pm 0,1$ & $8,7 \pm 0,1$ & $8,3 \pm 0,1$ & $8,3 \pm 0,1$ & $8,3 \pm 0,1$ \\
\hline SR - FSC1 & $7,9 \pm 0,1$ & $9,0 \pm 0,1$ & $8,9 \pm 0,1$ & $8,2 \pm 0,1$ & $8,3 \pm 0,1$ & $7,7 \pm 0,2$ & $8,3 \pm 0,4$ & $7,9 \pm 0,1$ \\
\hline SR - FSC1+P & $7,7 \pm 0,1$ & $9,0 \pm 0,1$ & $9,0 \pm 0,1$ & $8,8 \pm 0,1$ & $8,9 \pm 0,1$ & $7,6 \pm 0,1$ & $7,9 \pm 0,4$ & $8,1 \pm 0,4$ \\
\hline SR - FSC2 & $8,8 \pm 0,1$ & $8,8 \pm 0,1$ & $9,0 \pm 0,1$ & $8,9 \pm 0,1$ & $8,7 \pm 0,2$ & $8,0 \pm 0,1$ & $7,9 \pm 0,2$ & $7,5 \pm 0,1$ \\
\hline \multirow[t]{2}{*}{$\mathrm{SR}-\mathrm{FSC} 2+\mathrm{P}$} & $8,4 \pm 0,1$ & $8,8 \pm 0,2$ & $9,1 \pm 0,1$ & $9,0 \pm 0,1$ & $8,7 \pm 0,1$ & $7,9 \pm 0,1$ & $7,5 \pm 0,1$ & $7,7 \pm 0,1$ \\
\hline & \multicolumn{8}{|c|}{$\mathrm{CE}\left(\mathrm{mS} \mathrm{m} \mathrm{m}^{-1}\right)$} \\
\hline CR - FSC1 & $73 \pm 4,6$ & $73 \pm 2,5$ & $52 \pm 3,6$ & $40 \pm 2,9$ & $59 \pm 2,9$ & $46 \pm 1,9$ & $67 \pm 3,9$ & $77 \pm 5,8$ \\
\hline $\mathrm{CR}-\mathrm{FSC} 1+\mathrm{P}$ & $68 \pm 4,6$ & $68 \pm 4,5$ & $52 \pm 1,7$ & $45 \pm 3,1$ & $52 \pm 4,3$ & $53 \pm 1,4$ & $77 \pm 5,1$ & $85 \pm 2,4$ \\
\hline CR - FSC2 & $85 \pm 4,9$ & $85 \pm 2,2$ & $77 \pm 3,4$ & $57 \pm 0,6$ & $70 \pm 4,7$ & $58 \pm 1,7$ & $80 \pm 4,8$ & $82 \pm 3,4$ \\
\hline $\mathrm{CR}-\mathrm{FSC} 2+\mathrm{P}$ & $77 \pm 4,9$ & $77 \pm 7,5$ & $75 \pm 1,1$ & $71 \pm 6,5$ & $81 \pm 4,1$ & $64 \pm 1,1$ & $75 \pm 4,3$ & $88 \pm 3,3$ \\
\hline SR - FSC1 & $73 \pm 4,6$ & $51 \pm 2,4$ & $46 \pm 3,6$ & $68 \pm 2,7$ & $57 \pm 2,1$ & $73 \pm 6,8$ & $64 \pm 4,5$ & $68 \pm 5,8$ \\
\hline SR - FSC1+P & $68 \pm 4,6$ & $50 \pm 2,4$ & $47 \pm 5,4$ & $51 \pm 2,5$ & $45 \pm 2,0$ & $41 \pm 6,9$ & $57 \pm 4,0$ & $65 \pm 3,4$ \\
\hline SR - FSC2 & $85 \pm 4,9$ & $75 \pm 5,9$ & $57 \pm 7,0$ & $69 \pm 3,7$ & $58 \pm 2,3$ & $78 \pm 4,6$ & $91 \pm 3,6$ & $100 \pm 4,8$ \\
\hline SR - FSC2+P & $77 \pm 4,9$ & $81 \pm 8,2$ & $63 \pm 1,1$ & $76 \pm 3,2$ & $75 \pm 3,3$ & $98 \pm 8,4$ & $110 \pm 1,8$ & $89 \pm 12,9$ \\
\hline
\end{tabular}



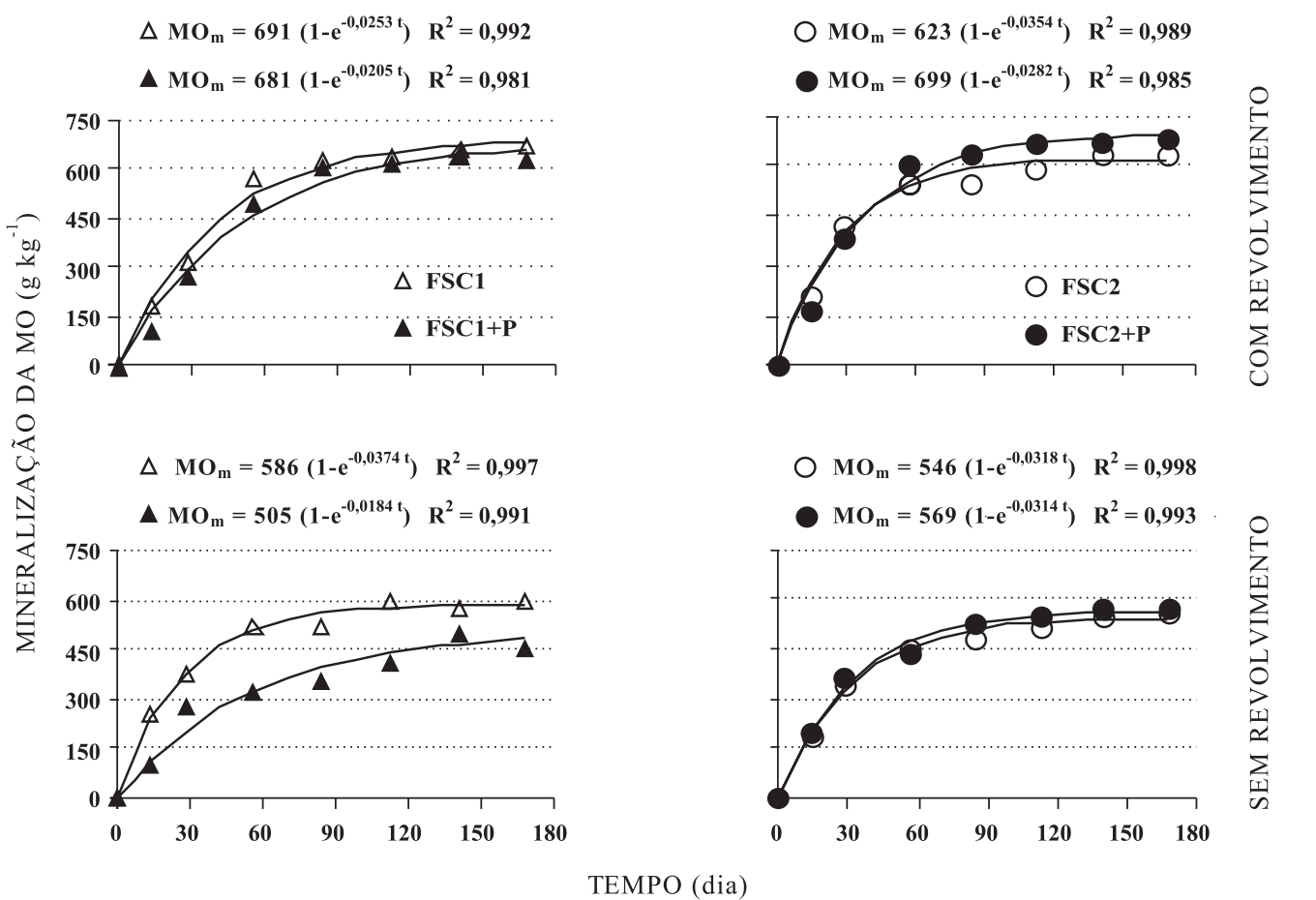

Figura 2. Perdas de matéria orgânica das pilhas de compostagem da fração sólida do chorume (FSC). As pilhas incluíram diferentes origens (FSC1 e FSC2, recolhidos de fossa aberta e fechada, respectivamente), com e sem revolvimento, sem e com palha $(\mathrm{P})$.

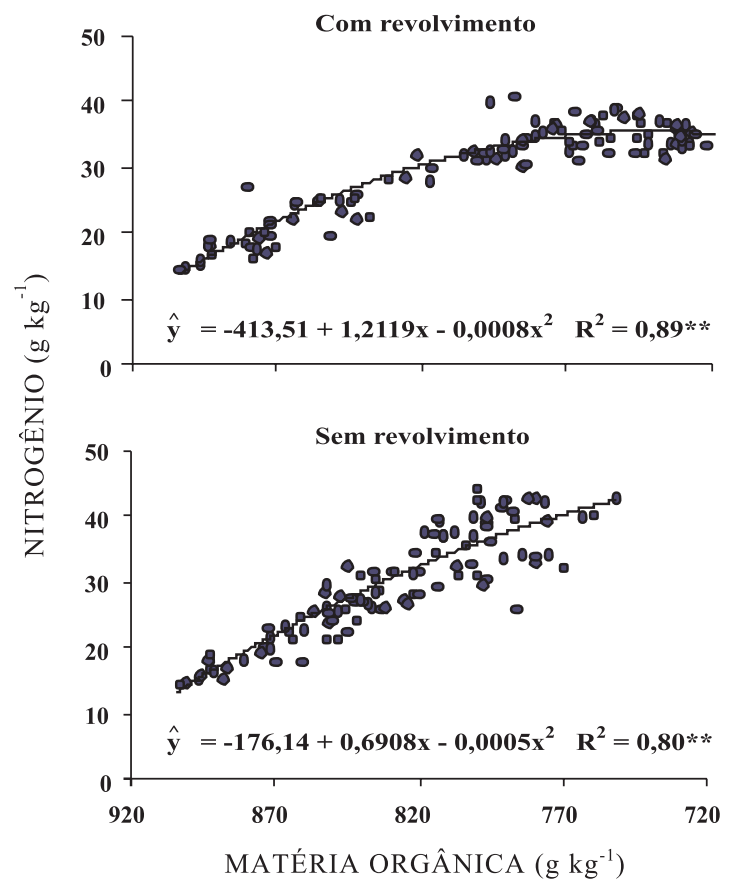

Figura 3. Relação entre o teor de nitrogênio e o de matéria orgânica nas amostras das pilhas de compostagem da fração sólida do chorume.

No início da compostagem da FSC, o teor de N$\mathrm{NO}_{3}{ }^{-}$foi muito baixo, 15 e $23 \mathrm{mg} \mathrm{kg}^{-1}$, respectivamente, para a FSC1 e para a FSC2. O teor de $\mathrm{N}_{-} \mathrm{NH}_{4}{ }^{+}$foi elevado, mas inferior na FSC1 (515 $\left.\mathrm{mg} \mathrm{kg}^{-1}\right)$ em comparação com a FSC2 $\left(1.096 \mathrm{mg} \mathrm{kg}^{-1}\right)$. Duas semanas após o início da compostagem, o teor de $\mathrm{N}-\mathrm{NH}_{4}{ }^{+}$diminuiu acentuadamente em todas as pilhas, sendo muito baixo no final da fase mais ativa da compostagem (Figura 5). Entre dois e quatro meses após o início da compostagem, o teor de $\mathrm{N}$ nítrico ultrapassou o de $\mathrm{N}$ amoniacal em todas as pilhas. A nitrificação iniciou-se mais cedo nas pilhas da FSC1 com revolvimento, em comparação com as mesmas pilhas sem revolvimento. No final da compostagem, o teor de $\mathrm{N}^{-\mathrm{NO}_{3}}{ }_{3}$ nas pilhas estáticas foi mais elevado para a FSC2, em comparação com a FSC1.

\section{DISCUSSÃO}

A temperatura da FSC durante a compostagem evoluiu como previsível (Inbar et al., 1993; Ros et al., 2006), aumentando inicialmente em consequência da degradação da MO facilmente disponível e, à medida que a MO ficou menos disponível, o calor produzido pelas reações exotérmicas associadas ao metabolismo microbiano diminuiu. A fase termófila da compostagem prolongou-se por 1,5 a 2 meses nas pilhas com a FSC1. Posteriormente, a temperatura diminuiu até atingir valores próximos da temperatura ambiente, 3 a 4 meses após o início da compostagem, indicando diminuição da atividade microbiana, sem o risco de um período mais prolongado de maturação do composto. 

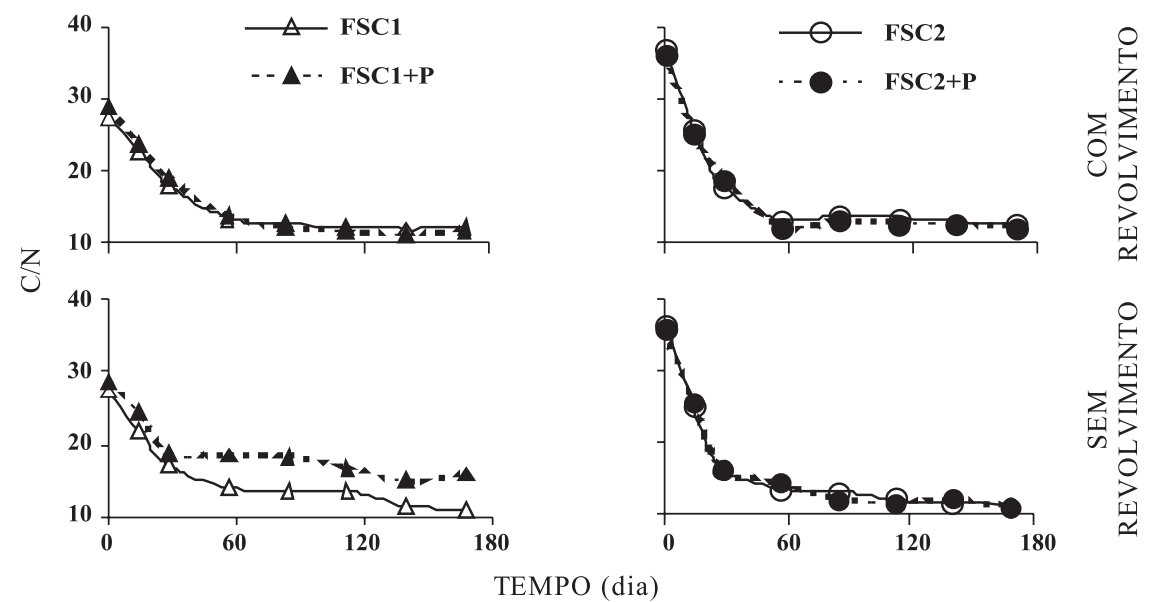

Figura 4. Evolução da relação $\mathrm{C} / \mathrm{N}$ das pilhas em função do tempo de compostagem (dias) da fração sólida do chorume (FSC). As pilhas incluíram diferentes origens (FSC1 e FSC2, recolhidos de fossa aberta e fechada, respectivamente), com revolvimento e sem revolvimento, sem e com palha (P).
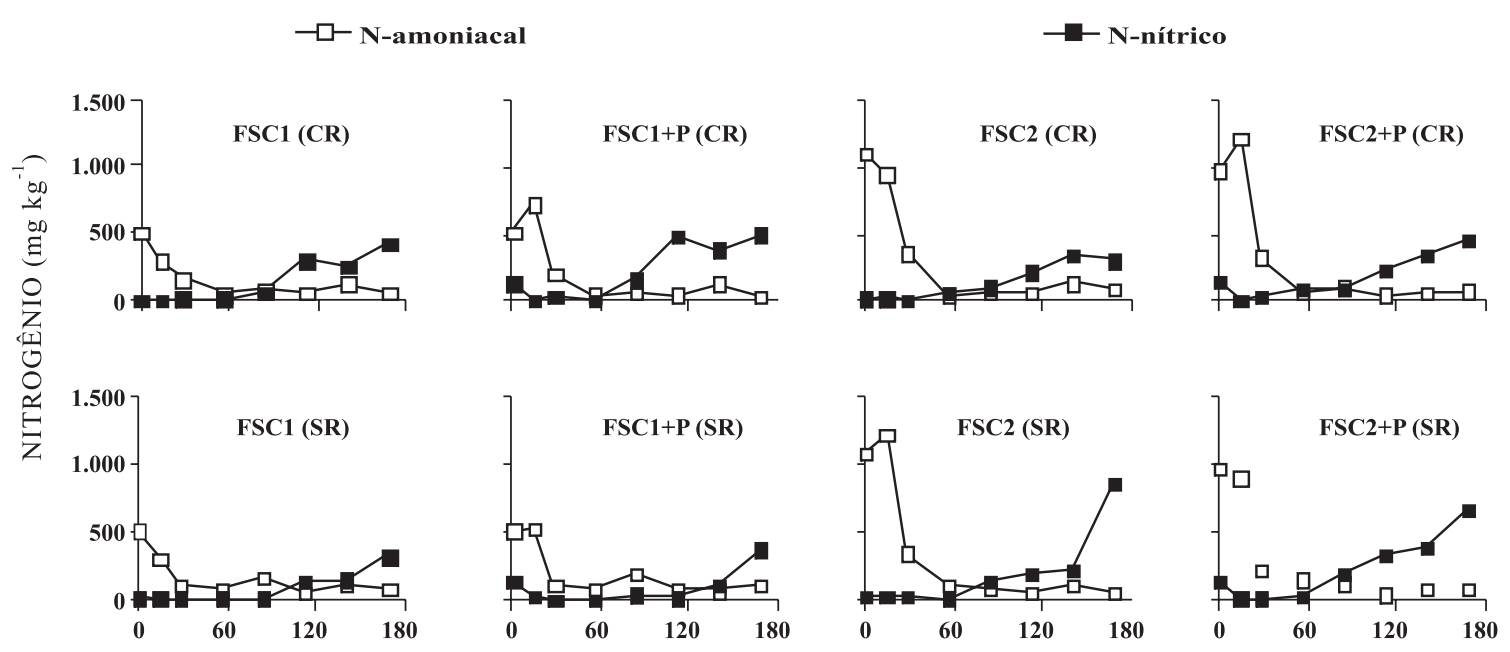

TEMPO DE COMPOSTAGEM (dia)

Figura 5. Evolução do teor de nitrogênio mineral durante a compostagem da fração sólida do chorume (FSC) com revolvimento (CR) e sem revolvimento (SR). As pilhas incluíram diferentes origens (FSC1 e FSC2, recolhidos de fossa aberta e fechada, respectivamente), sem e com palha (P).

Enquanto a compostagem da FSC1 pode ter garantido a higienização do composto e a eliminação das sementes viáveis de infestantes, o mesmo poderá não ter acontecido durante a compostagem da FSC2, já que esta decorreu quase sempre com temperaturas mesófilas. O aumento da temperatura da FSC1, em comparação com a de FSC2, poderá ser explicada pelo maior arejamento da FSC1, associado ao menor rendimento da separação. A mistura com palha favoreceu o aparecimento de temperaturas mais elevadas no início da compostagem em todas as pilhas, bem como o aparecimento de temperaturas superiores a $60{ }^{\circ} \mathrm{C}$ nas pilhas estáticas da FSC1. Isso é devido provavelmente, ao fato de o fluxo de $\mathrm{O}_{2}$ para o interior das pilhas ser facilitado pela palha.
A umidade nas pilhas foi geralmente superior ao valor máximo (70 \%) sugerido por Inbar et al. (1988), acima do qual o material fica saturado de água e a atividade microbiana diminui por falta de $\mathrm{O}_{2}$. Na generalidade das pilhas, a umidade da FSC não diminuiu durante a compostagem porque a evaporação de água não compensou a produção de água resultante do processo de mineralização da matéria orgânica e a diminuição da matéria seca.

De acordo com Tchobanoglous et al. (1993), o valor de $\mathrm{pH}$ não deveria exceder 8,5 para minimizar as perdas de $\mathrm{NH}_{3}$, fato que aconteceu em diversas ocasiões nessas pilhas. No final da compostagem, o valor de $\mathrm{pH}$ variou entre 7,2 e 8,5 , indicando que a 
concentração de bicarbonatos (muito utilizados na alimentação animal) não foi suficientemente reduzida para que o composto fosse utilizado como substrato de propagação (Cáceres et al., 2006) e que a compostagem da FSC poderá se beneficiar com a mistura com outros materiais que possuam um valor de $\mathrm{pH}$ baixo, para diminuir as perdas de $\mathrm{NH}_{3}$ (Raviv et al., 2004).

$\mathrm{O}$ fato de a MO ter sido mineralizada a uma taxa superior nas pilhas com revolvimento em comparação com as pilhas estáticas pode ser explicado pelo aumento do arejamento, o que permitiu aumentar a concentração de $\mathrm{O}_{2}$ no interior das pilhas, aumentando assim a atividade metabólica dos microrganismos e a velocidade do processo de compostagem. As perdas de MO durante a compostagem da FSC foram semelhantes às perdas de 46 a $62 \%$, referidas por Eghball et al. (1997), durante a compostagem de estrume bovino. O teor de MO diminuiu de forma muito acentuada nos primeiros dois meses de compostagem e, posteriormente, estabilizou-se nas pilhas com revolvimento três meses após o início da compostagem e após 4 meses nas pilhas estáticas, indicando forte redução nos materiais orgânicos facilmente biodegradáveis, em comparação com a MO mais estabilizada.

A relação $\mathrm{C} / \mathrm{N}$ tem sido utilizada como indicador do grau de decomposição dos materiais orgânicos (Witter \& Lopez-Real, 1987). Diversos autores apontaram valores da relação C/N inferiores a 20 como indicadores de uma maturação aceitável (Poincelot, 1974; Cardenas \& Wang, 1980). Zucconi \& Bertoldi (1987), por exemplo, sugeriram que uma diminuição entre valores de 35-40 no início para valores de 18-20 ou inferiores, no final da compostagem, implicavam estado avançado de maturação. Nesses ensaios, a relação $\mathrm{C} / \mathrm{N}$ pouco variou entre as oito modalidades de compostagem consideradas e diminuiu até valores geralmente inferiores a 13, parecendo indicar um elevado grau de estabilização do composto final (Zucconi \& Bertoldi, 1987; Bernal et al., 1998).

No final da compostagem, o teor de $\mathrm{N}$ total no composto foi, em todas as pilhas, muito superior ao teor de $\mathrm{N}$ da maioria dos compostos comerciais produzidos em climas quentes $\left(15 \mathrm{~g} \mathrm{~kg}^{-1}\right)$ e referido por Hadas \& Portnoy (1997). O teor de $\mathrm{N}$ nos compostos finais foi, em média, superior nas pilhas estáticas, fato que confirma a hipótese das perdas de N aumentarem com o revolvimento referida por outros autores (Hao \& Chang, 2001; Peigné \& Girardin, 2004). O revolvimento das pilhas de compostagem pode servir para fornecer $\mathrm{O}_{2}$ para o processo de decomposição, mas, realizado com demasiada freqüência, pode aumentar as emissões de $\mathrm{NH}_{3} \mathrm{e}$ reduzir o valor agronômico do produto final, por diminuir o teor de $\mathrm{N}$.

$\mathrm{O} \mathrm{N}$ mineral na FSC caracterizou-se por um elevado teor de $\mathrm{N}-\mathrm{NH}_{4}{ }^{+}$e baixo teor de $\mathrm{N}-\mathrm{NO}_{3}{ }^{-}$no início da compostagem. Os riscos de lixiviação foram nulos ou muito reduzidos, quer durante a fase termófila da compostagem, porque o teor de nitrato era muito baixo; quer durante a maturação, porque, apesar de o teor de $\mathrm{NO}_{3}{ }^{-}$ter aumentado consideravelmente, não se verificou uma umidade que provocasse sua lixiviação.

O aumento de temperatura no início da compostagem influenciou o equilíbrio $\mathrm{NH}_{4}{ }^{+}: \mathrm{NH}_{3}$ no sentido da produção de $\mathrm{NH}_{3}$, aumentando sua transferência para a atmosfera. $\mathrm{O}$ pH elevado também aumenta o equilíbrio $\mathrm{NH}_{4}{ }^{+}: \mathrm{NH}_{3}$ no sentido da produção de $\mathrm{NH}_{3}$. Por estas razões, o valor de $\mathrm{pH}$ elevado e a temperatura elevada durante o processo de compostagem podem ter condicionado o balanço $\mathrm{NH}_{3} /$ $\mathrm{NH}_{4}{ }^{+}$e a emissão de $\mathrm{NH}_{3}$. No entanto, a volatilização de $\mathrm{NH}_{3}$ pode ter sido limitada pela imobilização do $\mathrm{N}$ nos microrganismos decompositores, particularmente na FSC2, na qual a relação $\mathrm{C} / \mathrm{N}$ foi superior no início da compostagem. $\mathrm{O}$ baixo teor de $\mathrm{N}-\mathrm{NH}_{4}{ }^{+}$, combinado com o aumento do teor de $\mathrm{N}-\mathrm{NO}_{3}{ }^{-}$no final do período de compostagem e com a baixa relação $\mathrm{C} / \mathrm{N}$ e a descida de temperatura, sugerem que o composto estava estabilizado aos 168 dias de compostagem.

\section{CONCLUSÕES}

1. A incorporação de palha nas pilhas de compostagem da fração sólida do chorume bovino (FSC) e o aumento do teor de matéria seca da FSC contribuíram para elevar a temperatura das pilhas e, conseqüentemente, para garantir a higienização dos compostos.

2. A baixa temperatura, a baixa relação $\mathrm{C} / \mathrm{N}$ e o baixo teor de $\mathrm{NH}_{4}{ }^{+}$em combinação com o aumento do teor de $\mathrm{NO}_{3}{ }^{-}$dos compostos finais indicaram que estes estavam estabilizados.

3. As características químicas de $\mathrm{pH}$ ligeiramente alcalino, a baixa condutividade eléctrica e os elevados teores de $\mathrm{N}$ e MO dos compostos da FSC sugerem que estes podem ter utilização como corretivos orgânicos do solo, com benefícios agronômicos e ambientais.

\section{AGRADECIMENTOS}

Este projeto foi apoiado pelo projeto AGRO 794, financiado pela União Europeia e pelo Instituto Nacional de Investigação Agrária de Portugal (INIAP).

\section{LITERATURA CITADA}

BERNAL, M.P.; PAREDES, C.; SÁNCHEZ-MONEDERO, M.A. \& CEGARRA, J. Maturity and stability parameters of compost prepared with a wide range of organic wastes. Biores. Technol., 63:91-99, 1998. 
BRITO, L.M.; MOURÃO, I.; TRINDADE, H. \& COUTINHO, J. Fate of $\mathrm{N}$ during composting of the solid fraction of dairy cattle slurry. In: SCHRODER, J.J. \& NEETESON, J.J., eds. $\mathrm{N}$ management in agrosystems in relation to the water framework directive. Dordrecht, Plant Research International, 2006. p.136-139.

CÁCERES, R.; FLOTATS, X. \& MARFÀ, O. Changes in the chemical and physicochemical properties of the solid fraction of cattle slurry during composting using different aeration strategies. Waste Manag., 26:1081-1091, 2006.

CARDENAS, R.R. \& WANG, L.K. Composting process. In: HANDBOOK of environmental engineering. New York, The Human Press, 1980. v.2. p.269-327.

EUROPEAN COMMITTEE FOR STANDARDIZATION - CEN Soil improvers and growing media. Brunnels, 1999.

CHADWICK, D.R. Emissions of ammonia, nitrous oxide and methane from cattle manure heaps: Effect of compaction and covering. Atmosph. Environ., 39:787-799, 2005.

EGHBALL, B.; POWER, J.F.; GILLEY, J.E. \& DORAN, J.W. Nutrient, carbon, and mass loss during composting of beef cattle feedlot manure. J. Environ. Qual., 26:189. 193, 1997.

FORD, M. \& FLEMING, R. Mechanical solid-liquid separation of livestock manure. Literature review. In: REPORT to Ontario Pork, case study 7 - Screw Press. Ridgetown College, University of Guelph, 2002. 49p.

FUKUMOTO, Y.; OSADA, T.; HANAJIMA, D. \& HAGA, K. Patterns and quantities of $\mathrm{NH}_{3}, \mathrm{~N}_{2} \mathrm{O}$ and $\mathrm{CH}_{4}$ emissions during swine manure composting without forced aerationeffect of compost pile scale. Biores. Technol., 89:109-114, 2003.

GOLUEKE, C.G. Biological reclamation of soil wastes. Emmaus, Rodale Press, 1977. p.137-142.

GONÇALVES, M.S. \& BAPTISTA, M. Proposta de regulamentação sobre qualidade do composto para utilização na agricultura. Laboratório Químico Agrícola Rebelo da Silva. Lisboa, INIA, MADRP, 2001. 14p.

HADAS, A. \& PORTNOY, R. Rates of decomposition in soil and release of available nitrogen from cattle manure and municipal waste compost. Compost Sci. Util., 53:48-54, 1997.

HANSEN, M.N.; HENRIKSEN, K. \& SOMMER, S.G. Observations of production and emission of greenhouse gases and ammonia during storage of solids separated from pig slurry: Effects of covering. Atmosph. Environ., 40:4172-4181, 2006

HAO, X. \& CHANG, C. Gaseous NO, $\mathrm{NO}_{2}$, and $\mathrm{NH}_{3}$ loss during cattle feedlot manure composting. Phyton-Annales Rei Botanicae, 41:81-93, 2001.

HELLMANN, B.; ZELLES, L.; PALOJARVI, A. \& BAI, Q. Emission of climate-relevant trace gases and succession of microbial communities during open-windrow composting. Appl. Environ. Microbiol., 63:1011-1018, 1997.
HOUBA, V.J.G.; van der LEE, J.J. \& NOVOZAMSKY, I. Soil analysis procedures - other procedures, part 5B. 6.ed. Wageningen, Wageningen Agricultural University, 1995.

HUANG, G.F.; WU, Q.T.; WONG, J.W.C. \& NAGAR, B.B. Transformation of organic matter during co-composting of pig manure with sawdust. Biores. Technol., 97:1834$1842,2006$.

INBAR, Y.; CHEN, Y.; HADAR, Y. \& VERDONCK, O. Composting of agricultural wastes for their use as container media: Simulation of the composting process. Biol. Wastes 26:247-259, 1988.

INBAR, Y.; HADAR, Y. \& CHEN, Y. Recycling of cattle manure, the composting process and characterization of maturity. J. Environ. Qual., 22:857-863, 1993.

MILLER, F.C.; HARPER, E.R. \& MACALULEY, B.J. Field examination of temperature and oxygen relationships in mushroom composting stacks-consideration of stack oxygenation based on utilization and supply. Austr. J. Exp. Agric., 29:741-750, 1989.

MØLLER, H.B.; LUND, I. \& SOMMER, S.G. Solid-liquid separation of livestock slurry: Efficiency and cost. Biores. Technol., 74:223-229, 2000.

MYERS, R.J.K. Temperature effects on ammonification and nitrification in a tropical soil. Soil Biol. Biochem., 7:83-98, 1975.

OENEMA, O.; BANNINK, A.; SOMMER, S.G. \& VELHOF, G.L. Gaseous nitrogen emissions from livestock farming systems. In: FOLLETT, R.F. \& HARTFIELD, J.L., eds. Nitrogen in the environment: Sources, problems, and management. Amsterdam, Elsevier, 2001. p.255-290.

PAREDES, C.; ROIG, A.; BERNAL, M.P.; SÁNCHEZMONEDERO, M.A. \& CEGARRA, J. Evolution of organic matter and nitrogen during co-composting of olive mill wastewater with solid organic wastes. Biol. Fert. Soils, 20:226-236, 2000 .

PEIGNÉ, J. \& GIRARDIN, P. Environmental impacts on farm scale composting practices. Water, Air Soil Poll., 153:45$68,2004$.

POINCELOT, R.P. A scientific examination of the principles and pratice of composting. Compost. Sci., 15:24-31, 1974.

RAVIV, M.; MEDINA, S.; KRASNOVSKY, A. \& ZIADNA, H. Organic matter and nitrogen conservation in manure compost for organic agriculture. Compost Sci. Util., 12:6$10,2004$.

ROS, M.; GARCIA, C. \& HERNÁNDEZ, T. A full-scale study of treatment of pig slurry by composting:Kinetic changes in chemical and microbial properties. Waste Manag., 26:1108-1118, 2006.

SOMMER, S.G. Effect of composting on nutrient loss and nitrogen availability of cattle deep litter. Eur. J. Agric., 14, 123-133, 2001.

SOMMER, S.G. \& MOLLER, H.B. Emission of greenhouse gases during composting of deep litter from pig production - effect of straw content. J. Agric. Sci., 134:327-335, 2000. 
STENTIFORD, E.T. Composting control, principles and practice. In: DEBERTOLDI, M.; SEQUI, P.; LEMMES, B. \& PAPI, T., eds. The science of composting. London, Chapman Hall, 1996. p.49-59.

TCHOBANOGLOUS, G.; THEISEN, H. \& VIGIL, S.A. Integrated solid waste management, engineering principles and management issues. New York, McGrawHill, 1993. 978p. (Water Resources and Environmental Engineering)

TIQUIA, S.M.; TAM, N.F.Y. \& HODGKISS, I.J. Effects of turning frequency on composting of spent pig-manure sawdust litter. Biores. Technol., 62:37-42, 1997.

TIQUIA, S.M. \& TAM, N.F.Y. Fate of nitrogen during composting of chicken litter. Environ. Poll., 110:535-541, 2000.
WEBB, J.; CHADWICK, D. \& ELLIS, S. Will storing farmyard manure in compact anaerobic heaps be a simple and effective means of reducing ammonia emissions? In: SANGIORGI, F., ed. Technology transfer. Paris, Cemagref, 2001. p.161-166.

WITTER, E. \& LOPEZ-REAL, J.M. Monitoring the composting process using parameters of compost stability. In: DE BERTOLDI, M.; FERRANTI, M.P.; L'HERMITE, P. \& ZUCCONI, F., eds. Compost: Quality and use. London, Elsevier Applied Science, 1987. p.351-358.

ZUCCONI, F. \& BERTOLDI, M. Composts specifications for the production and characterization of composts from municipal solid waste. In: DE BERTOLDI, M.; FERRANTI, M.P.; L'HERMITE, P. \& ZUCCONI, F., eds. Compost: Quality and use. London, Elsevier Applied Science, 1987. p.30-50. 